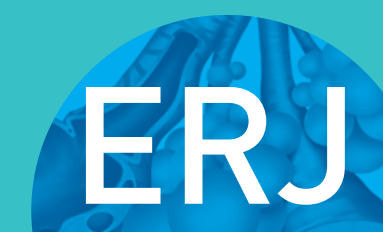

open research
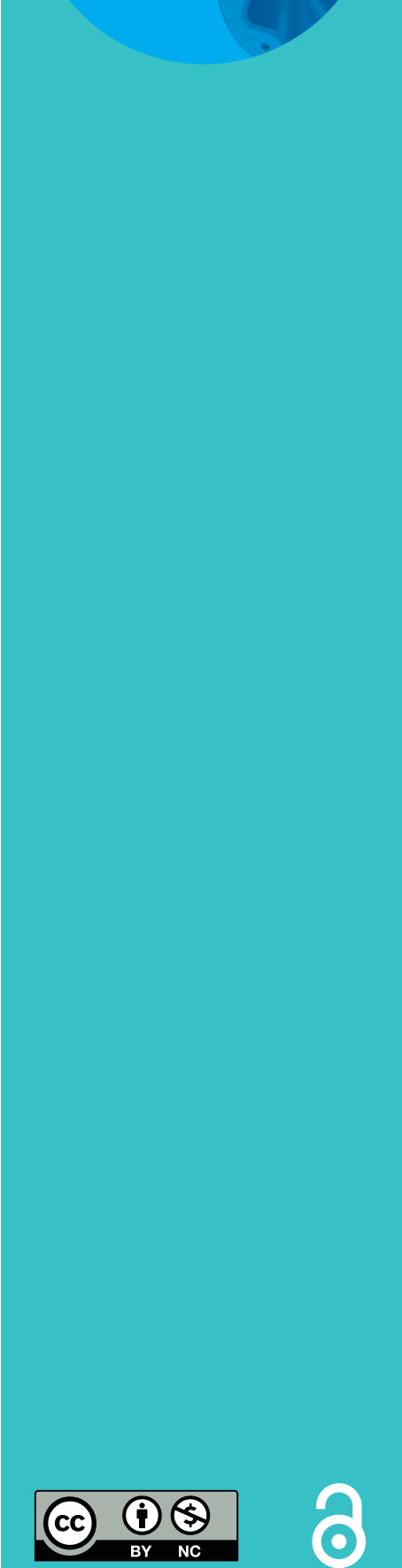

\section{Distinct immune regulatory receptor profiles linked to altered monocyte subsets in sarcoidosis}

\author{
Simon D. Fraser ${ }^{1}$, Michael G. Crooks ${ }^{1}$, Paul M. Kaye ${ }^{2}$ and Simon P. Hart (1) ${ }^{1}$
}

Affiliations: ${ }^{1}$ Respiratory Research Group, Hull York Medical School, Castle Hill Hospital, Cottingham, UK. ${ }^{2}$ York Biomedical Research Institute, Hull York Medical School, University of York, York, UK.

Correspondence: Simon P. Hart, Respiratory Research Group, Hull York Medical School, Castle Hill Hospital, Castle Road, Cottingham, HU16 5JQ, UK. E-mail: s.hartahull.ac.uk

\section{ABSTRACT}

Background: In sarcoidosis, blood monocytes, circulating precursors of granuloma macrophages, display enhanced inflammatory cytokine production, reduced expression of the regulatory (inhibitory) receptor CD200R, and altered subsets defined by CD14 and CD16. Regulatory receptors serve to dampen monocyte and macrophage inflammatory responses. We investigated the relationship between monocyte subsets and regulatory receptor expression in sarcoidosis.

Methods: Multiparameter flow cytometry was used to perform detailed analyses of cell surface regulatory molecules on freshly isolated blood immune cells from patients with chronic pulmonary sarcoidosis and age-matched healthy controls.

Results: 25 patients with chronic pulmonary sarcoidosis (median duration of disease 22 months) who were not taking oral corticosteroids or other immunomodulators were recruited. Nonclassical monocytes were expanded in sarcoidosis and exhibited significantly lower expression of regulatory receptors CD200R, signal regulatory protein- $\alpha$ and CD47 than classical or intermediate monocytes. In sarcoidosis, all three monocyte subsets had significantly reduced CD200R and CD47 expression compared with healthy controls. A dichotomous distribution of CD200R was seen on classical and intermediate monocytes in the sarcoidosis population, with 14 out of $25(56 \%)$ sarcoidosis patients having a CD200R ${ }^{\text {low }}$ phenotype and 11 out of $25(44 \%)$ having a CD200R ${ }^{\text {high }}$ phenotype. These distinct sarcoidosis monocyte phenotypes remained consistent over time.

Conclusions: Nonclassical monocytes, which are expanded in sarcoidosis, express very low levels of regulatory receptors. Two distinct and persistent phenotypes of CD200R expression in classical and intermediate monocytes could be evaluated as sarcoidosis biomarkers.

@ERSpublications

Nonclassical monocytes, which are expanded in sarcoidosis, express very low levels of regulatory receptors. Two distinct and persistent phenotypes of CD200R expression in classical and intermediate monocytes could be evaluated as sarcoidosis biomarkers. https://bit.ly/2W0idAX

Cite this article as: Fraser SD, Crooks MG, Kaye PM, et al. Distinct immune regulatory receptor profiles linked to altered monocyte subsets in sarcoidosis. ERJ Open Res 2021; 7: 00804-2020 [https:// doi.org/10.1183/23120541.00804-2020].

This article has supplementary material available from openres.ersjournals.com

Received: 30 Oct 2020 | Accepted after revision: 4 Dec 2020

Copyright $\odot$ ERS 2021. This article is open access and distributed under the terms of the Creative Commons Attribution Non-Commercial Licence 4.0. 


\section{Introduction}

Sarcoidosis is characterised by granulomas composed of activated monocyte-derived macrophages [1]. Studying circulating blood monocytes offers a window to granuloma macrophage biology and, unlike sarcoid tissue biopsies, allows repeated sampling. Research in sarcoidosis has identified alterations in circulating monocyte populations defined by expression of surface receptors CD14 (lipopolysaccharide co-receptor) and CD16 (low-affinity receptor for IgG). Reduced classical (CD14 $\left.{ }^{++} / \mathrm{CD} 16^{-}\right)$monocytes and increased CD16-expressing nonclassical $\left(\mathrm{CD} 14^{+} / \mathrm{CD} 16^{++}\right)$or intermediate $\left(\mathrm{CD} 14^{++} / \mathrm{CD} 16^{+}\right)$monocytes have been reported [2-5]. Enhanced production of inflammatory cytokines by sarcoidosis monocytes was associated with reduced expression of the regulatory receptor CD200R [2], which serves to dampen immune responses. CD200R deficiency in animals leads to enhanced production of tumour necrosis factor (TNF) $[6,7]$, a critical driver of granulomatous inflammation. Therefore, relative lack of CD200R could contribute to the exaggerated inflammation seen in chronic sarcoidosis. The relationship between sarcoidosis monocyte subsets and expression of CD200R and other regulatory receptors is unknown.

\section{Methods}

\section{Subjects}

Patients with chronic pulmonary sarcoidosis were recruited from the sarcoidosis clinic at Hull University Teaching Hospitals NHS Trust (Hull, UK). Patients were diagnosed by a specialist interstitial lung disease physician according to international guidance [8]. Exclusion criteria included current tobacco smoking or therapy with oral corticosteroids or other immunomodulatory drugs, which could independently affect monocyte phenotype. Healthy controls were age- and sex-matched with the patient cohort. Controls were volunteers without sarcoidosis or significant lung disease who were nonsmokers and were not taking oral corticosteroids or immunosuppressant medication. Detailed medical histories were not obtained from healthy controls nor were pulmonary function tests or blood investigations performed. A statistical power calculation was not performed for this exploratory study. Ethical approval was obtained from the Yorkshire and Humber Research Ethics Committee (REC 16/YH/0118) and participants gave written informed consent.

\section{Immune cell phenotyping}

Blood was collected in sodium heparin tubes (BD, Oxford, UK) and processing was started within $1 \mathrm{~h}$. Peripheral blood mononuclear cells (PBMCs) were isolated using density gradient centrifugation [2]. For immunophenotyping, live unfixed PBMCs were stained with saturating combinations of fluorescent antibodies (supplementary tables S1 and S2) and multiparameter flow cytometry was used to analyse antibody binding, allowing discrimination of surface receptor expression on cellular subsets. Details of the immunophenotyping procedures can be found in the supplementary material.

\section{TABLE 1 Demographic and clinical profiles of patients with sarcoidosis and healthy controls}

\begin{tabular}{|c|c|c|}
\hline & Sarcoidosis & Controls \\
\hline Patients & 25 & 12 \\
\hline Age years & 53 (38-73) & $51(30-67)$ \\
\hline \multicolumn{3}{|l|}{ Sex } \\
\hline Male & 15 & 7 \\
\hline Female & 10 & 5 \\
\hline Time since diagnosis months & $22(0-193)$ & \\
\hline \multicolumn{3}{|l|}{ Scadding chest radiograph stage } \\
\hline 0 & 3 & \\
\hline 1 & 5 & \\
\hline 2 & 12 & \\
\hline 3 & 5 & \\
\hline Angiotensin converting enzyme U. $\mathrm{L}^{-1 \#}$ & $94(9-440)$ & \\
\hline Blood lymphocyte count $\times 10^{9} \mathrm{~L}^{-1 \pi}$ & $0.86(0.29-2.03)$ & \\
\hline $\mathrm{FEV}_{1} \%$ pred & $93(54-126)$ & \\
\hline FVC \% pred & $95(64-129)$ & \\
\hline $\mathrm{FEV}_{1} / \mathrm{FVC}$ & $0.81(0.49-0.91)$ & \\
\hline
\end{tabular}


Immunohistochemistry

The procedure for staining tissue sections for CD200L and CD200R was adapted from CAwKwELL et al. [9]. Antibodies were a polyclonal goat CD200L antibody (AF2724; R\&D Systems, Abingdon, UK), normal goat IgG control (AB-108-C; R\&D Systems), mouse monoclonal IgG1 anti-human CD200R (OX108, MCA 2282T; AbD Serotec/Bio-Rad, Kidlington, UK) and mouse monoclonal control IgG1 antibody (MOPC-21, 400101; Biolegend, London, UK).

\section{Statistical analyses}

Statistical significance was determined using the Mann-Whitney U-test or Kruskal-Wallis test for nonparametric distributions and the t-test, one-way ANOVA or two-way ANOVA for parametric distributions. Multiple comparisons underwent Šídák or Tukey's or post hoc analyses as appropriate. Pearson's Chi-squared or Fisher's exact tests were used to examine associations between categorical variables. Analyses were performed using Prism (GraphPad, La Jolla, CA, USA).

\section{Results}

\section{Patient demographics}

25 treatment-naive patients with chronic pulmonary sarcoidosis were recruited. Baseline characteristics of the patients are presented in table 1. All participants were of White ethnicity, reflecting the local population.

\section{Nonclassical monocytes are increased in sarcoidosis and express low levels of CD200R}

Sarcoidosis patients had significantly increased proportions of nonclassical monocytes and significantly lower proportions of classical monocytes compared with controls (figure 1). Nonclassical monocytes expressed significantly lower levels (10-15\%) of CD200R than intermediate or classical monocytes in both sarcoidosis patients and controls $(\mathrm{p}<0.0001)$ (supplementary figure $\mathrm{S} 1$ ).
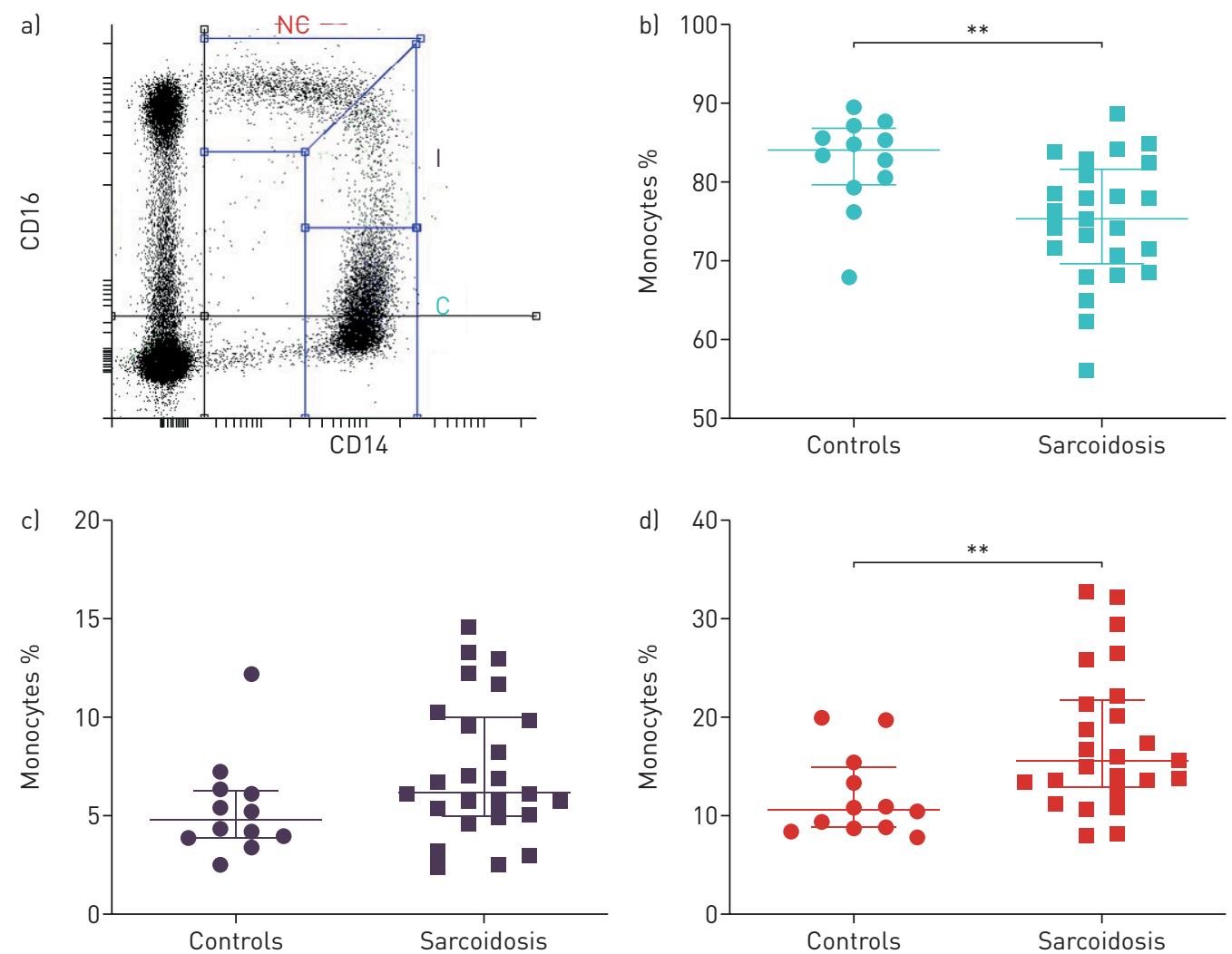

FIGURE 1 Patients with sarcoidosis have increased nonclassical monocytes and reduced classical monocytes compared with controls. Flow cytometric analysis of peripheral blood mononuclear cells (PBMCs) was used to define monocyte subsets based on expression of CD14 and CD16. Ungated PBMCs are shown here for

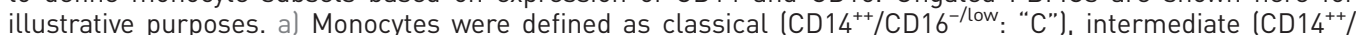
CD16 : "I") or nonclassical (CD14 $/$ CD16 ${ }^{++}$: "NC"). b-d) Proportions of b) classical, c) intermediate and d) nonclassical monocyte subsets within total blood monocytes for patients with sarcoidosis $(n=25)$ and healthy controls $(n=12)$. Individual data points are presented along with median and interquartile range. **: $p<0.01$ (Mann-Whitney U-test). 
Dichotomous populations of monocyte CD200R expression in sarcoidosis

Patients with sarcoidosis had significantly lower expression of CD200R on all monocyte subsets compared with controls (figure 2). In the sarcoidosis cohort, CD200R expression on classical and intermediate monocytes was not normally distributed. Normal quantile-quantile plots (supplementary figure S2) confirmed bimodal populations with inflection points delineating CD200R ${ }^{\text {high }}$ from CD200R ${ }^{\text {low }}$ at a geometric mean fluorescence intensity (GMFI) of $\sim 300$.

To establish whether CD200R expression on monocytes was stable or varied over time, patients were tracked every 2 months for up to 1 year in a longitudinal study. The two distinct sarcoidosis patient populations with $\mathrm{CD} 200 \mathrm{R}^{\text {high }}$ and $\mathrm{CD} 200 \mathrm{R}^{\text {low }}$ classical and intermediate monocyte subsets maintained their phenotypes throughout follow-up (figure 3). No individual switched between populations over time.

11 patients (44\%) exhibited high CD200R expression on classical and intermediate monocytes (median (range) GMFI 505 (359-664)) and 14 (56\%) had low CD200R expression (median (range) GMFI 157 (70-276)). Most control subjects had high CD200R expression on classical and intermediate monocytes, but we also observed a low classical monocyte CD200R phenotype in three of the 12 healthy controls.

$\mathrm{CD} 200 \mathrm{R}^{\text {high }}$ or $\mathrm{CD} 200 \mathrm{R}^{\text {low }}$ monocyte phenotypes in sarcoidosis patients were not associated with age, sex, extent of chest radiograph changes, lung function, serum angiotensin converting enzyme activity, C-reactive protein or blood cell counts (table 2). Comorbidities and medication use were evenly distributed across the two monocyte phenotypes (data not shown).

The effect of systemic corticosteroid therapy on monocyte subsets and CD200R expression was examined in three patients who progressed to need treatment with oral prednisolone during follow-up. Oral prednisolone therapy for 2 months $\left(20 \mathrm{mg} \mathrm{day}^{-1}\right)$ tended to normalise monocyte subsets (figure 4$)$, with post-treatment increases in classical monocytes and decreases in nonclassical monocytes. Statistical significance could not be demonstrated with only three pairs of data. In contrast, prednisolone therapy had no demonstrable effect on monocyte CD200R expression (figure 4).
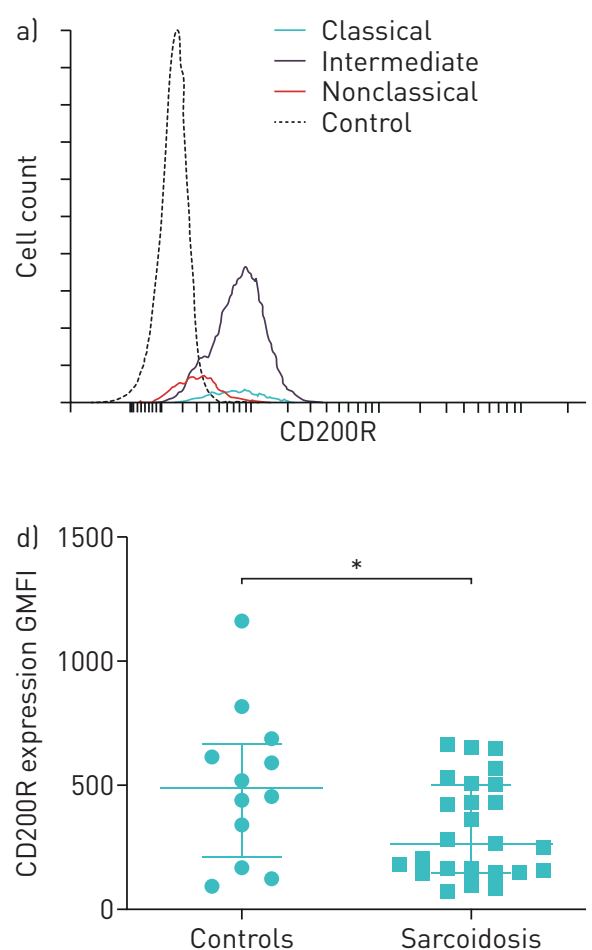
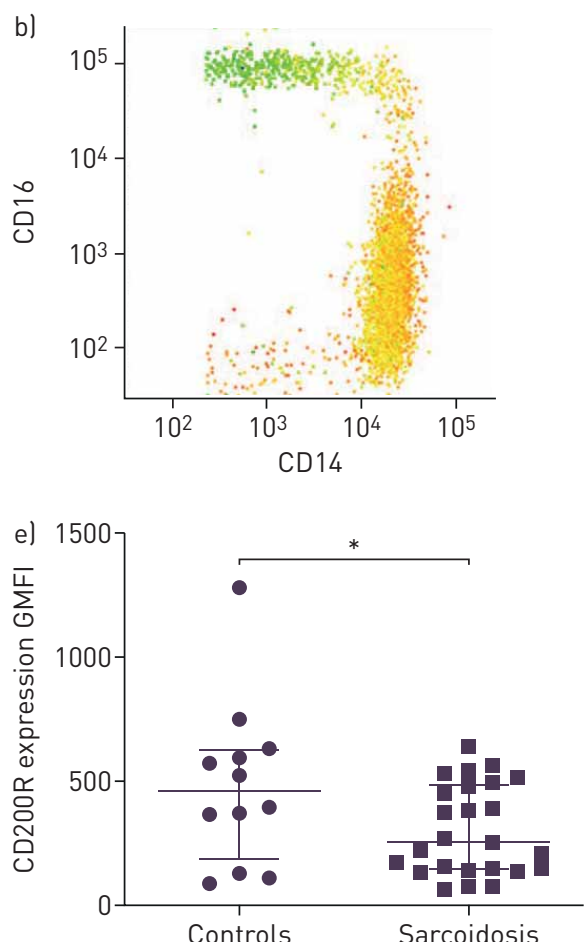
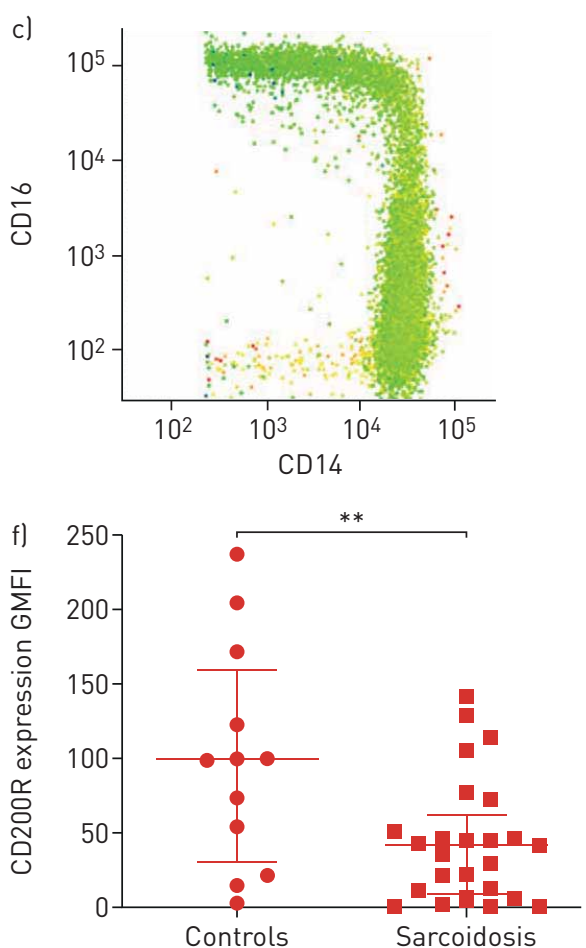

FIGURE 2 Nonclassical monocytes express low levels of CD200R and expression of CD200R is lower on all monocyte subsets in patients with sarcoidosis compared with healthy controls. GMFI: geometric mean fluorescence intensity. a) Representative flow cytometry histograms from a healthy control subject comparing CD200R expression on classical, intermediate and nonclassical monocytes compared with isotype control antibody. b, c) Representative heat-map dot plots from b) a healthy control subject demonstrating low CD200R on nonclassical monocytes and c) a patient with sarcoidosis showing reduced CD200R expression on sarcoidosis monocyte subsets compared with the healthy control. On these pseudo-colour plots, orange indicates higher CD200R expression and green indicates lower CD200R expression. d-f) Expression of CD200R on d) classical, e) intermediate and f) nonclassical monocyte subsets in sarcoidosis patients and controls. Note the small $y$-axis scale for nonclassical monocytes. Individual data points are presented along with median and interquartile range. *: p<0.05; **: $\mathrm{p}<0.01$ (Mann-Whitney U-test). 

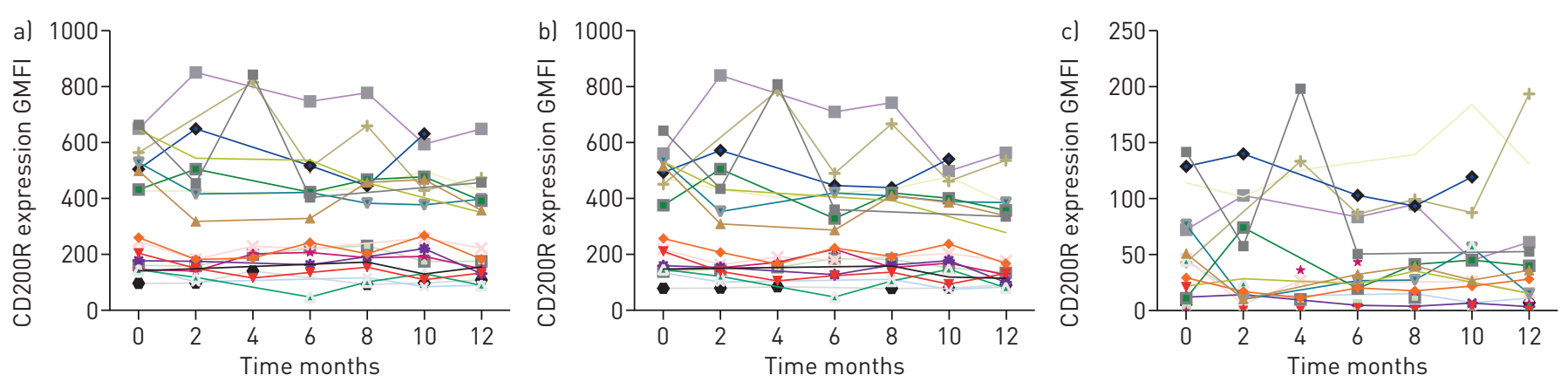

FIGURE 3 CD200R expression on sarcoidosis classical and intermediate monocytes is dichotomous and consistent over time. GMFI: geometric mean fluorescence intensity. Patients with sarcoidosis in a longitudinal cohort ( $n=19$ ) underwent serial measurements of $C D 200 R$ expression on a) classical, b) intermediate and c) nonclassical monocyte subsets. Each patient is presented by a different coloured line.

\section{Expression of regulatory receptors signal regulatory protein- $\alpha, C D 47$ and interleukin-10R on sarcoidosis monocytes}

CD200R is one of several inhibitory receptors that dampen inflammatory responses in monocytes, so we studied whether other regulatory molecules were differentially expressed by monocyte subsets. We focused on molecules that, like CD200R, are known to regulate monocyte inflammatory responses, namely signal regulatory protein- $\alpha$ (SIRP- $\alpha$, which binds CD47 and surfactant proteins), CD47 (the ligand for SIRP- $\alpha$ and receptor for thrombospondin-1 that delivers an anti-phagocytic signal) and interleukin (IL)-10R (the receptor for IL-10, an anti-inflammatory cytokine). Expression of CD47 and SIRP- $\alpha$ was significantly lower on nonclassical monocytes compared with classical or intermediate monocytes, whereas expression of IL-10R was generally low but was highest on intermediate monocytes (figure 5 and supplementary figure S1).

TABLE 2 Characteristics of sarcoidosis patients stratified by monocyte CD200R phenotype

\begin{tabular}{|c|c|c|c|}
\hline & $\mathrm{CD}^{200 R^{\text {low }}}$ & CD200R high & p-value \\
\hline Patients & 14 & 11 & \\
\hline Scadding chest radiograph stage & & & 0.265 \\
\hline 0 & 1 & 2 & \\
\hline 1 & 4 & 1 & \\
\hline 2 & 5 & 7 & \\
\hline 4 & 4 & 1 & \\
\hline Angiotensin converting enzyme U. $\mathrm{L}^{-1}$ & $92(52-136)$ & 104 (74-155) & 0.695 \\
\hline C-reactive protein $\mathrm{mg} \cdot \mathrm{L}^{-1}$ & $2.0(1.4-5.5)$ & $2.8(0.6-9.6)$ & 0.217 \\
\hline Age years ${ }^{\#}$ & $53.5(40-73)$ & $50.0(38-67)$ & 1.00 \\
\hline FEV $_{1} \%$ pred & $99(69-110)$ & $92(82-113)$ & 0.428 \\
\hline FVC $\%$ pred & $103(76-115)$ & $90(82-114)$ & 0.428 \\
\hline $\mathrm{FEV}_{1} / \mathrm{FVC}$ & $0.79(0.75-0.84)$ & $0.83(0.76-0.86)$ & 0.238 \\
\hline Time since diagnosis months & $20(9-51)$ & $31(10-73)$ & 0.695 \\
\hline Sex & & & 1.00 \\
\hline Male & 8 & 7 & \\
\hline Female & 6 & 4 & \\
\hline White blood cell count $\times 10^{9} \mathrm{~L}^{-1}$ & $5.4(4.15-6.20)$ & $4.3(3.50-5.60)$ & 0.196 \\
\hline Lymphocyte count $\times 10^{9} \mathrm{~L}^{-1}$ & $0.93(0.74-1.41)$ & $0.74(0.62-1.02)$ & 0.279 \\
\hline Monocyte count $\times 10^{9} \mathrm{~L}^{-1}$ & $0.55(0.44-0.72)$ & $0.42(0.24-0.70)$ & 0.252 \\
\hline Neutrophil count $\times 10^{9} \mathrm{~L}^{-1}$ & $3.42(2.70-3.98)$ & $2.70(2.33-3.97)$ & 0.336 \\
\hline Eosinophil count $\times 10^{9} \mathrm{~L}^{-1}$ & $0.15(0.13-0.23)$ & $0.20(0.08-0.21)$ & 0.797 \\
\hline T-lymphocyte count $\times 10^{9} \mathrm{~L}^{-1}$ & $0.61(0.36-0.91)$ & $0.48(0.33-0.63)$ & 0.403 \\
\hline $\mathrm{CD4}^{+}$T-lymphocyte count $\times 10^{9} \mathrm{~L}^{-1}$ & $0.36(0.22-0.70)$ & $0.30(0.16-0.45)$ & 0.596 \\
\hline CD8 ${ }^{+}$T-lymphocyte count $\times 10^{9} \mathrm{~L}^{-1}$ & $0.20(0.11-0.40)$ & $0.17(0.09-0.21)$ & 0.290 \\
\hline B-lymphocyte count $\times 10^{9} \mathrm{~L}^{-1}$ & $0.16(0.07-0.21)$ & $0.14(0.08-0.23)$ & 0.499 \\
\hline Natural killer cell count $\times 10^{9} \mathrm{~L}^{-1}$ & $0.17(0.12-0.22)$ & $0.15(0.09-0.26)$ & 0.450 \\
\hline \multicolumn{4}{|c|}{$\begin{array}{l}\text { Data are presented as } \mathrm{n} \text { or median (interquartile range), unless otherwise stated. } \mathrm{FEV}_{1} \text { : forced expiratory } \\
\text { volume in } 1 \mathrm{~s} ; \mathrm{FVC} \text { : forced vital capacity. "\#: median (range). Statistical comparisons by nonparametric } \\
\text { independent samples median test or Pearson's Chi-squared test. }\end{array}$} \\
\hline
\end{tabular}



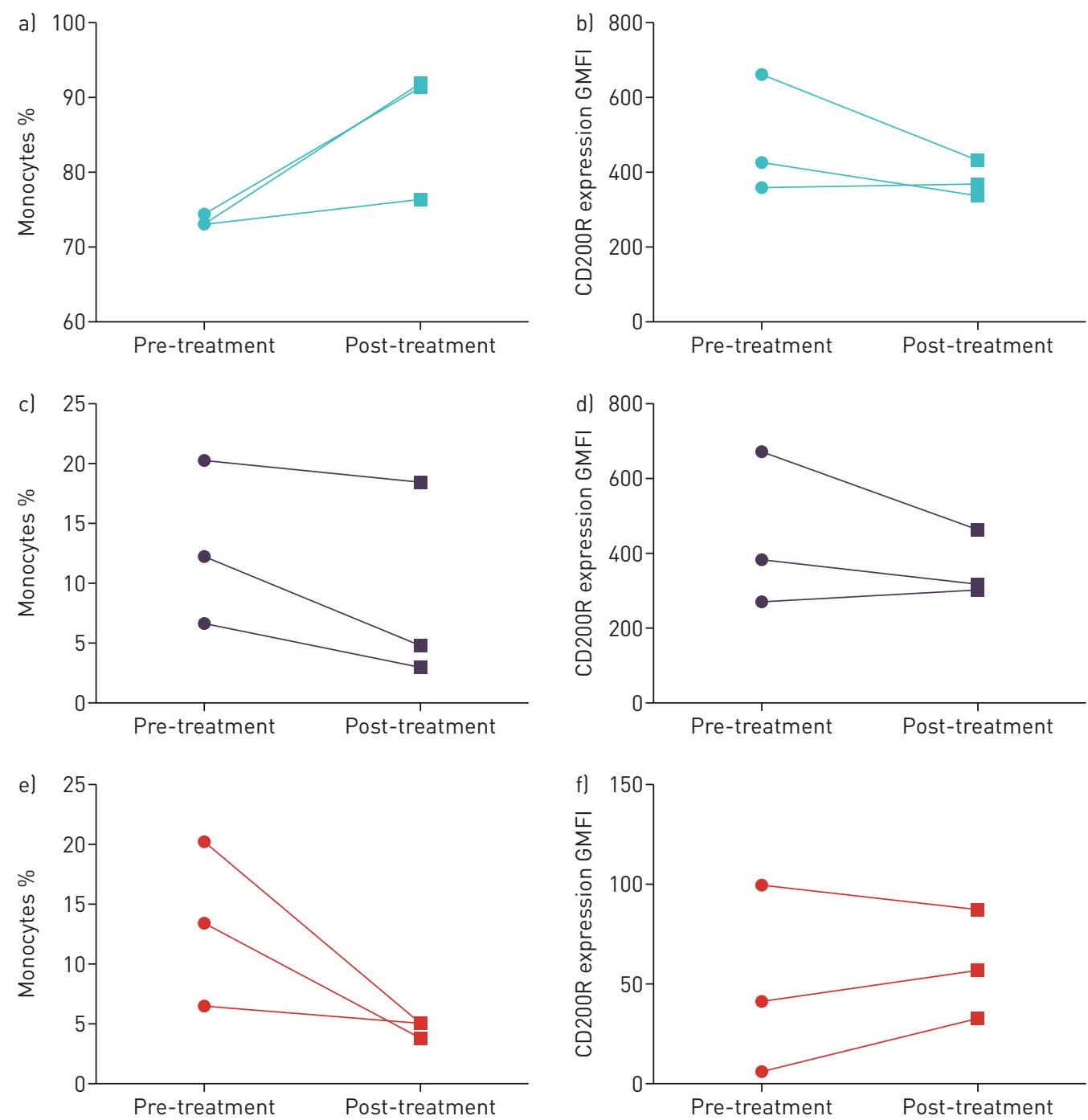

FIGURE 4 Oral corticosteroid therapy for sarcoidosis normalises monocyte subsets but has no effect on monocyte CD200R expression. GMFI: geometric mean fluorescence intensity. Pre- and 2-month post-steroid treatment data for three sarcoidosis patients who were treated with oral prednisolone $20 \mathrm{mg} \cdot \mathrm{day}^{-1}$. $a, c, e)$ Percentages of classical, intermediate and nonclassical monocytes, respectively. b, d, f) CD200R expression on the respective monocyte subsets.

Comparing patients with sarcoidosis and healthy controls demonstrated significantly reduced expression of CD47 on all monocyte subsets in sarcoidosis (figure 5). IL-10R expression was reduced only on intermediate monocytes in patients with sarcoidosis compared with controls, but not on other subsets. SIRP- $\alpha$ expression was similar in controls and sarcoidosis patients.

\section{Distribution of CD200R and CD200L in sarcoidosis granulomas}

To gain a preliminary view of how macrophage regulatory receptors may interact with ligands within a sarcoidosis granuloma, we stained sarcoidosis biopsies for the receptor-ligand pairing of CD200R and CD200L. Figure 6 shows the spatial relationship between CD200R-expressing granuloma macrophages and neighbouring CD200L-positive lymphocytes and fibroblasts.

\section{Discussion}

Understanding the cellular and molecular drivers of persistent and progressive sarcoidosis will be an important step towards identifying new targets for treatment. Chronic, nonresolving sarcoidosis is characterised immunologically by heightened inflammatory responses of tissue granuloma macrophages and their precursors, circulating blood monocytes [2, 5, 10-14]. Cell surface regulatory receptors such as CD200R serve to dampen inflammatory responses in monocytes and macrophages, including reducing 

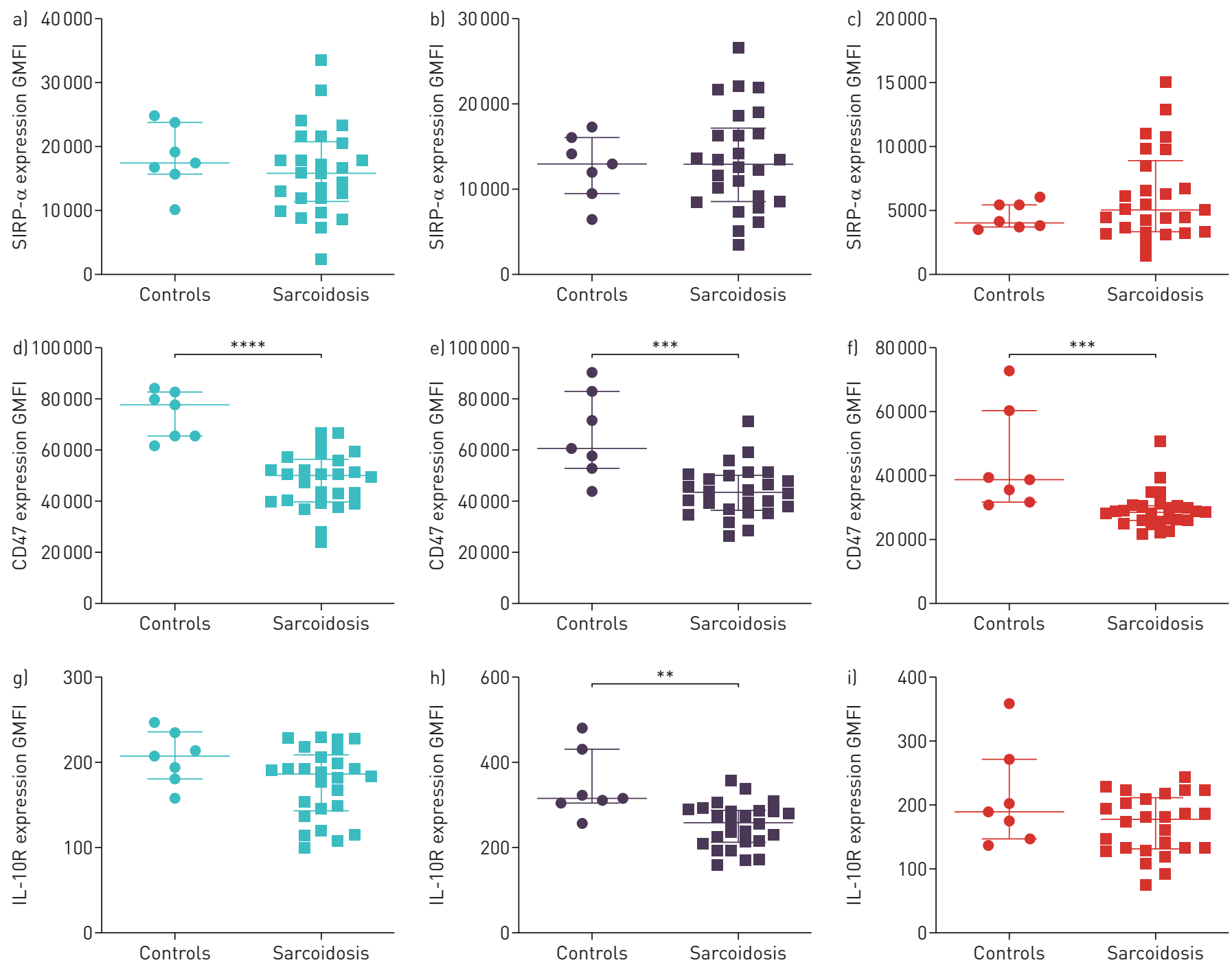

FIGURE 5 Monocyte subsets have distinct expression profiles of signal regulatory protein- $\alpha$ (SIRP- $\alpha$ ), CD47 and interleukin (IL)-10R in sarcoidosis and healthy controls. Expression of $a-c)$ SIRP- $\alpha, d-f$ ) CD47 and g-i) IL-10R on a, d, g) classical, b, e, h) intermediate and c, f, i) nonclassical monocyte subsets in patients with sarcoidosis $(n=25)$ and healthy controls $(n=7)$. Individual data points are presented along with median and interquartile range. ${ }^{* *}$ : $p<0.01 ;{ }^{* * *}: p<0.001 ;{ }^{* * *}: p<0.0001$ (Mann-Whitney U-test).

production of TNF, which is a key cytokine driving granuloma formation and persistence $[15,16]$. Altered regulatory receptor expression is a potential mechanism contributing to persistent and progressive granulomatous inflammation in sarcoidosis.

We provide the first description that monocyte subsets have distinct expression profiles of regulatory receptors. Nonclassical monocytes exhibited much lower expression of CD200R, SIRP- $\alpha$ and CD47 than classical or intermediate monocytes. Moreover, in sarcoidosis all monocyte subsets had reduced CD200R and CD47 expression compared with healthy controls, providing an additional explanation for the overall reduced regulatory receptor profile. However, there is not a global reduction of all regulatory molecules in sarcoidosis, since expression levels of SIRP- $\alpha$ or IL-10R were similar to controls.

In health, most $(\sim 90 \%)$ monocytes are classical $\mathrm{CD} 14^{++} / \mathrm{CD} 16^{-}$cells [17]. The remainder comprise CD16-positive nonclassical monocytes which exhibit higher stimulated TNF production [18] and a low frequency intermediate $\left(\mathrm{CD} 14^{++} / \mathrm{CD} 6^{+}\right)$monocyte population. A developmental relationship between monocyte subsets has been described, with sequential increase in first intermediate then nonclassical monocytes [19]. Distinguishing nonclassical from intermediate monocytes within the CD16-positive population can be challenging $[4,17,20]$, so it is difficult to compare studies directly. In the present study, we identified a clear increase in nonclassical monocytes in sarcoidosis, whereas previous studies reported that intermediate monocytes are increased $[2,5]$. Aside from differences between cohorts, the current 

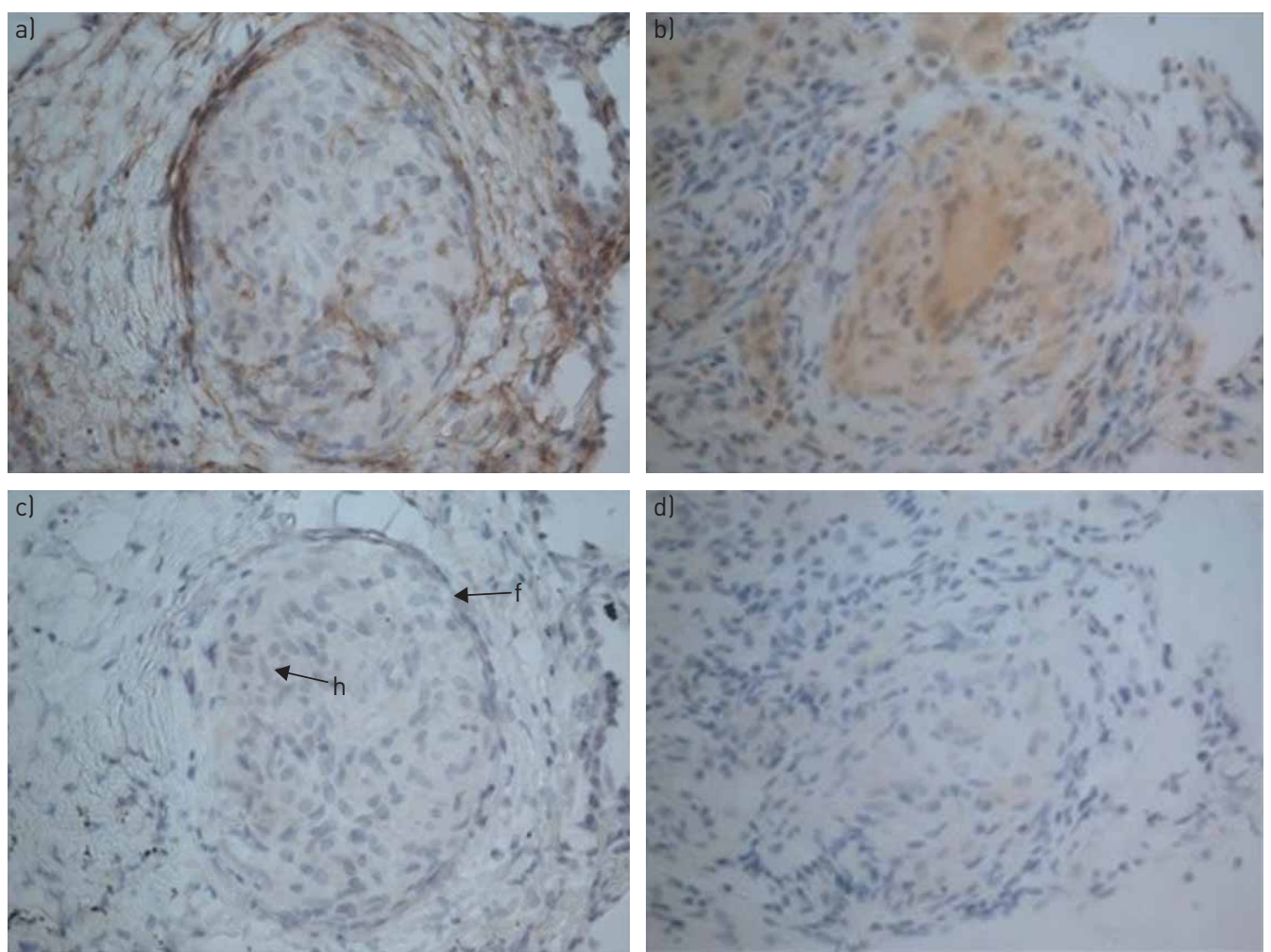

FIGURE 6 Distribution of the regulatory receptor CD200R and its ligand CD200L in sarcoidosis granulomas: transbronchial lung biopsy samples from two patients with sarcoidosis stained for a) CD200L and b) CD200R, with respective isotype control antibody staining in c) and d). f: fibroblasts; h: histiocytes (macrophages). Original magnification $\times 100$.

findings can be explained by increased refinement of monocyte gating. By utilising a fluorophore with a high stain index for CD16, adopting an angled gate between intermediate and nonclassical monocyte populations, and employing a robust gating protocol across all populations, the present study presents a more precise separation of monocyte subsets than we previously used. Although we did not refine discrimination of intermediate and nonclassical monocytes with additional markers such as 6-sulfo LacNAc (slan) [4], we have shown that CD200R expression clearly distinguishes nonclassical and intermediate monocytes, supporting the validity of our gating strategy and suggesting that CD200R has both functional and phenotypic importance and could be incorporated in future studies. Moreover, CD200R could be used in future studies as a marker of nonclassical monocytes.

Distribution of CD200R expression on classical and intermediate monocytes was dichotomous, such that patients with sarcoidosis could be divided into $\mathrm{CD} 200 \mathrm{R}^{\text {high }}$ and $\mathrm{CD} 200 \mathrm{R}^{\text {low }}$ groups. The stability of this monocyte CD200R phenotype over time suggests two distinct and consistent phenotypes of sarcoidosis. In our previous study, there was a dichotomous distribution of CD200R on total monocytes in sarcoidosis, whereas all of the 17 healthy controls had a CD200R ${ }^{\text {high }}$ phenotype. In the present study, three of the 12 controls exhibited a CD200R ${ }^{\text {low }}$ phenotype on classical and intermediate monocytes. This difference between the studies may be explained by careful age matching of the control with patient groups in the present study. We cannot rule out the possibility that the ethnically White population studied comprises two subpopulations, one with high and one with low CD200R expression, and that individuals with low expression are over-represented within a sarcoid patient group.

Immunohistochemistry showed CD200R-expressing macrophages concentrated in the central core of the sarcoidosis granuloma, surrounded by CD200L-expressing lymphocytes and fibroblasts. We anticipate that within the dynamic milieu of a sarcoidosis granuloma, CD200R on macrophages will be ligated by CD200L on neighbouring cells as they migrate and interact with each other.

We provide the first description of significantly reduced regulatory receptor expression on nonclassical monocytes. Expansion of these nonclassical monocytes in sarcoidosis, together with reduced levels of specific regulatory molecules $(\mathrm{CD} 200 \mathrm{R}$ and $\mathrm{CD} 47)$ on all sarcoidosis monocyte subsets compared with controls, will favour heightened inflammatory responses. This sarcoidosis monocyte phenotype could be assessed as a prognostic or therapeutic biomarker in future studies. 
Author contributions: S.D. Fraser performed the experiments and analysed the data. M.G. Crooks and S.P. Hart recruited the patients. All the authors designed the study, wrote the funding application, critiqued the data, and revised and approved the manuscript.

Support statement: This work was funded by a research grant from SarcoidosisUK and the British Lung Foundation (SRG15-15). P.M. Kaye is supported by a Wellcome Trust Senior Investigator Award (WT104726). Funding information for this article has been deposited with the Crossref Funder Registry.

Data availability: Individual subject data will not be shared.

Conflict of interest: S.D. Fraser reports grants from SarcoidosisUK/British Lung Foundation, during the conduct of the study. M.G. Crooks reports grants from SarcoidosisUK/British Lung Foundation, during the conduct of the study. P.M. Kaye reports grants from SarcoidosisUK/British Lung Foundation, during the conduct of the study. S.P. Hart reports grants from SarcoidosisUK/British Lung Foundation, during the conduct of the study.

\section{References}

1 Wilson JL, Mayr HK, Weichhart T. Metabolic programming of macrophages: implications in the pathogenesis of granulomatous disease. Front Immunol 2019; 10: 2265.

2 Fraser SD, Sadofsky LR, Kaye PM, et al. Reduced expression of monocyte CD200R is associated with enhanced proinflammatory cytokine production in sarcoidosis. Sci Rep 2016; 6: 38689.

3 Okamoto H, Mizuno K, Horio T. Circulating $\mathrm{CD}_{14}{ }^{+} \mathrm{CD} 16^{+}$monocytes are expanded in sarcoidosis patients. J Dermatol 2003; 30: 503-509.

4 Hofer TP, Zawada AM, Frankenberger M, et al. slan-defined subsets of CD16-positive monocytes: impact of granulomatous inflammation and M-CSF receptor mutation. Blood 2015; 126: 2601-2610.

5 Lepzien R, Rankin G, Pourazar J, et al. Mapping mononuclear phagocytes in blood, lungs, and lymph nodes of sarcoidosis patients. J Leukoc Biol 2019; 105: 797-807.

6 Boudakov I, Liu J, Fan N, et al. Mice lacking CD200R1 show absence of suppression of lipopolysaccharide-induced tumor necrosis factor-alpha and mixed leukocyte culture responses by CD200. Transplantation 2007; 84: 251-257.

7 Vaine CA, Soberman RJ. The CD200-CD200R1 inhibitory signaling pathway: immune regulation and host-pathogen interactions. Adv Immunol 2014; 121: 191-211.

8 American Thoracic Society, European Respiratory Society, World Association of Sarcoidosis and Other Granulomatous Disorders. Statement on sarcoidosis. Am J Respir Crit Care Med 1999; 160: 736-755.

9 Cawkwell L, Gray S, Murgatroyd H, et al. Choice of management strategy for colorectal cancer based on a diagnostic immunohistochemical test for defective mismatch repair. Gut 1999; 45: 409-415.

10 Yona S, Kim KW, Wolf Y, et al. Fate mapping reveals origins and dynamics of monocytes and tissue macrophages under homeostasis. Immunity 2013; 38: 79-91.

11 Talreja J, Farshi P, Alazizi A, et al. RNA-sequencing identifies novel pathways in sarcoidosis monocytes. Sci Rep 2017; 7: 2720.

12 Crawshaw A, Kendrick YR, McMichael AJ, et al. Abnormalities in iNKT cells are associated with impaired ability of monocytes to produce IL-10 and suppress T-cell proliferation in sarcoidosis. Eur J Immunol 2014; 44: $2165-2174$.

13 Romer FK, Christiansen SE, Kragballe K, et al. Studies of peripheral blood monocytes in pulmonary sarcoidosis Clin Exp Immunol 1984; 58: 357-363.

14 Mizuno K, Okamoto H, Horio T. Heightened ability of monocytes from sarcoidosis patients to form multi-nucleated giant cells in vitro by supernatants of concanavalin A-stimulated mononuclear cells. Clin Exp Immunol 2001; 126: 151-156.

15 Barclay AN, Wright GJ, Brooke G, et al. CD200 and membrane protein interactions in the control of myeloid cells. Trends Immunol 2002; 23: 285-290.

16 Manich G, Recasens M, Valente T, et al. Role of the CD200-CD200R axis during homeostasis and neuroinflammation. Neuroscience 2019; 405: 118-136.

17 Ziegler-Heitbrock L, Hofer TP. Toward a refined definition of monocyte subsets. Front Immunol 2013; 4: 23.

18 Belge KU, Dayyani F, Horelt A, et al. The proinflammatory CD $14^{+} \mathrm{CD} 16^{+} \mathrm{DR}^{++}$monocytes are a major source of TNF. J Immunol 2002; 168: 3536-3542.

19 Patel AA, Zhang Y, Fullerton JN, et al. The fate and lifespan of human monocyte subsets in steady state and systemic inflammation. J Exp Med 2017; 214: 1913-1923.

20 Ziegler-Heitbrock L, Ancuta P, Crowe S, et al. Nomenclature of monocytes and dendritic cells in blood. Blood 2010; 116: e74-e80. 